\title{
Muscle injury rate in professional football is higher in matches played within 5 days since the previous match: a 14-year prospective study with more than 130000 match observations
}

\author{
Håkan Bengtsson, 1,2 Jan Ekstrand, ${ }^{2,3}$ Markus Waldén, ${ }^{2,3}$ Martin Hägglund ${ }^{1,2}$
}

\begin{abstract}
Division of Physiotherapy, Department of Medical and Health Sciences, Linköping University, Linköping, Sweden ${ }^{2}$ Football Research Group, Linköping University, Linköping, Sweden

${ }^{3}$ Division of Community Medicine, Department of Medical and Health Sciences, Linköping University, Linköping, Sweden
\end{abstract}

\section{Correspondence to} Håkan Bengtsson, Division of Physiotherapy, Department of Medical and Health Sciences, Linköping University, Linköping, Sweden; info.frg@telia.com

Accepted 5 October 2017 Published Online First 3 November 2017
Check for updates

To cite: Bengtsson $\mathrm{H}$, Ekstrand J, Waldén $M$, et al. Br J Sports Med 2018:52:1116-1122.

\section{ABSTRACT}

Background The association between match congestion and injury rates in professional football has yielded conflicting results.

Aim To analyse associations between match congestion on an individual player level and injury rates during professional football matches.

Methods Data from a prospective cohort study of professional football with 133170 match observations were analysed with Poisson regressions. Associations between short-term match congestion, defined as number of days between two match exposures $(\leq 3,4$, 5, 6 and 7-10 days) and injury rates were analysed. To analyse the influence of long-term match congestion, defined as individual match exposure hours in the 30 days preceding a match, observations were categorised into three groups (low, $\leq 4.5$; medium, $>4.5$ to $\leq 7.5$; and high, $>7.5$ hours).

Results No differences in total match injury rates were found between the reference category ( $\leq 3$ days) and the other categories of short-term congestion. Muscle injury rates were significantly lower in matches preceded by 6 (rate ratio (RR) $0.79 ; 95 \% \mathrm{Cl} 0.65$ to 0.95 ) or $7-10$ days (RR $0.81 ; 95 \% \mathrm{Cl} 0.71$ to 0.93 ) compared with $\leq 3$ days since the last match exposure. No differences in total and muscle injury rates between the three long-term match congestion groups were found.

Conclusions In this study of male professional football players, there were no match congestion-related differences in total match injury rates, but muscle injury rates during matches were lower when players were given at least 6 days between their match exposures.

\section{INTRODUCTION}

Professional football carries a high risk of injury, with muscle injuries being the most common injury type. ${ }^{1}$ Muscular fatigue, defined as a decline in muscle performance associated with muscle activity, ${ }^{2}$ and signs of muscle damage may last more than 72 hours after a football match. ${ }^{3-6}$ Players may also experience mental fatigue following a match, ${ }^{7}$ with decreasing decision-making abilities as a possible consequence. ${ }^{8}$ Almost one-third of matches for top-level professional football teams are played within a 72 -hour interval. ${ }^{9}$ It is therefore plausible that players are not able to fully recover, physiologically and/or mentally, between matches.

Studies focused on short-term match congestion have shown that injury rates are higher if a team plays two matches separated by 4 or fewer compared with 6 or more days; ${ }^{910}$ in particular, muscle injury rates increase with fewer days between matches. ${ }^{9}$ Long-term match congestion has also been investigated in a few studies. While most of these studies have shown associations between match congestion and high injury rates, ${ }^{9} 1112$ one study reported no such association. ${ }^{13}$ Even though these studies have provided important initial information about the relationship between injuries and match congestion in professional football, they have been limited by either small sample sizes or by analysing match congestion at the team level. Furthermore, the combined effect of short-term and long-term match congestion on injury rates has not been investigated in football.

The aim of the present study was to analyse associations between match congestion and match injury rates at an individual player level. A secondary aim was to analyse whether associations between short-term match congestion and match injury rates are dependent on the player's long-term match congestion.

\section{METHODS}

This study was based on post hoc analyses of data gathered during 14 consecutive seasons from $2001 / 02$ to $2014 / 15$ in a prospective cohort study known as the UEFA Elite Club Injury Study. ${ }^{1}$ During this period, a total of 57 professional European teams from 16 countries were included, all participating in the highest division of their respective countries. All first team players from the included teams were invited to participate for a total of 2672 included players.

\section{Exposure and injury registration}

Individual exposure during matches was registered by a member of the technical staff. All time-loss injuries that occurred during these matches were registered on standard injury cards containing information about the occurrence and diagnosis of the injury (table 1). Injury cards and attendance records were reported to the study group monthly. The methodology of the exposure and injury registration has been described in detail previously. ${ }^{14}$

\section{Match congestion}

Two different variables were used to study the influence of short-term and long-term match congestion on injury rates during football matches. Shortterm match congestion was defined as the number 


\begin{tabular}{ll}
\hline $\begin{array}{l}\text { Table } 1 \\
\text { the study }\end{array}$ & Definitions of injury and match congestion variables used in \\
\hline Variable & Definition \\
\hline Time-loss injury & $\begin{array}{l}\text { Any physical complaint sustained by a player that } \\
\text { results from a football match and led to the player } \\
\text { being unable to take full part in future football } \\
\text { training or match play. }\end{array}$ \\
\hline Muscle injury & $\begin{array}{l}\text { A traumatic distraction or overuse injury to a } \\
\text { muscle. }\end{array}$ \\
\hline Short-term match congestion & $\begin{array}{l}\text { Number of days since the player's last recorded } \\
\text { match exposure prior to the observation. }\end{array}$ \\
\hline Long-term match congestion & $\begin{array}{l}\text { Total hours of match exposure that the player } \\
\text { had been exposed to in the 30 days prior to the } \\
\text { observation. }\end{array}$ \\
\hline
\end{tabular}

of days that had elapsed since the player's last recorded match exposure prior to an observation (eg, if a player played a match on Monday and then played another match on Thursday, 3 days had elapsed before the second match). Long-term match congestion was defined as the total hours of match exposure that the player had been exposed to in the past 30 days prior to an observation (table 1 ).

First team and national team competitive match observations (excluding first team friendly, reserve team and under-21 team matches) that were preceded by at least 30 days of reported exposure not interrupted by any occurring injury or absence due to injury were considered eligible for analysis. In addition, there should not have been more than 10 days between an observation and the players' last preceding match.

\section{Data analysis and statistics}

All statistical analyses were performed using the Statistical Package for the Social Sciences 23 (SPSS, Chicago, Illinois, USA). Injury rate was defined as the number of injuries per 1000 hours of match exposure. Match injury rates in different short-term match congestion groups (categorised as $\leq 3$ days, 4 days, 5 days, 6 days, and 7-10 days between matches) and rate ratios (RRs) between these groups were analysed with Poisson regressions using match exposure hours as an offset. In these analyses, short-term match congestion was included as the independent variable, with $\leq 3$ days serving as the reference category, while number of injuries and muscle injuries were used as dependent variables in separate analyses.

To investigate the influence of the duration of the previous match exposure, all observations were categorised in two groups $(<90 \mathrm{~min}$ or $\geq 90 \mathrm{~min}$ of exposure in previous match) which were analysed separately.

Additional analyses were made for those observations where the duration of the previous match exposure was $\geq 90 \mathrm{~min}$. First, to analyse associations between long-term match congestion and injury rates, observations were categorised in low ( $\leq 4.5$ hours), medium ( $>4.5$ to $\leq 7.5$ hours) or high ( $>7.5$ hours) match

Table 2 Characteristics of individual match observations categorised depending on short-term match congestion, and subgrouped based on the duration of the previous match exposure

\begin{tabular}{|c|c|c|c|c|c|}
\hline & \multicolumn{5}{|c|}{ Short-term match congestion (number of days between match exposures) } \\
\hline & $\leq 3$ days & 4 days & 5 days & 6 days & 7-10 days \\
\hline Observations, $\mathrm{n}$ (\% of total observations) & $35063(26 \%)$ & $33414(25 \%)$ & $12502(9 \%)$ & $13911(10 \%)$ & $38280(29 \%)$ \\
\hline Total match exposure, hours & 43529 & 41633 & 15753 & 17685 & 47833 \\
\hline Mean match exposure time/observation, minutes (SD) & $74.5(26.8)$ & $74.8(26.7)$ & $75.6(25.5)$ & $76.3(25.5)$ & $75.0(26.5)$ \\
\hline Mean long-term match congestion ${ }^{*}$, hours (SD) & $6.7(2.6)$ & $6.5(2.5)$ & $6.3(2.4)$ & $5.7(2.3)$ & $5.2(2.2)$ \\
\hline All injuries, $n$ & 1096 & 1040 & 383 & 420 & 1144 \\
\hline Total injury rate/1000 hours $(95 \% \mathrm{Cl})$ & 25.2 (23.7 to 26.7$)$ & $25.0(23.5$ to 26.5$)$ & 24.3 (22.0 to 26.9$)$ & 23.7 (21.6 to 26.1$)$ & 23.9 (22.6 to 25.3$)$ \\
\hline Total injury rate ratio $(95 \% \mathrm{Cl})$ & Reference group & 0.99 (0.91 to 1.08$)$ & 0.97 (0.86 to 1.08 ) & 0.94 (0.84 to 1.06$)$ & 0.95 (0.87 to 1.03$)$ \\
\hline Muscle injuries, $\mathrm{n}$ ( $\%$ of all injuries) & $435(40 \%)$ & $441(42 \%)$ & $157(41 \%)$ & $139(33 \%)$ & $389(34 \%)$ \\
\hline Muscle injury rate $/ 1000$ hours $(95 \% \mathrm{Cl})$ & $10.0(9.1$ to 11.0$)$ & $10.6(9.6$ to 11.6$)$ & $10.0(8.5$ to 11.7$)$ & $7.9(6.7$ to 9.3$)$ & $8.1(7.4$ to 9.0$)$ \\
\hline Muscle injury rate ratio $(95 \% \mathrm{Cl})$ & Reference group & $1.06(0.93$ to 1.21$)$ & $1.00(0.83$ to 1.20$)$ & 0.79 (0.65 to 0.95$)$ & 0.81 (0.71 to 0.93$)$ \\
\hline \multicolumn{6}{|l|}{$\geq 90$ min exposure in previous match } \\
\hline Total match exposure, hours & 29134 & 28498 & 10943 & 12578 & 33422 \\
\hline All injuries, $n$ & 721 & 683 & 277 & 289 & 760 \\
\hline Total injury rate/1000 hours $(95 \% \mathrm{Cl})$ & 24.7 (23.0 to 26.6$)$ & $24.0(22.2$ to 25.8$)$ & $25.3(22.5$ to 28.5$)$ & 23.0 (20.5 to 25.8$)$ & 22.7 (21.2 to 24.4$)$ \\
\hline Total injury rate ratio $(95 \% \mathrm{Cl})$ & Reference group & 0.97 (0.87 to 1.08$)$ & 1.02 (0.89 to 1.17$)$ & 0.93 (0.81 to 1.06$)$ & 0.92 (0.83 to 1.02$)$ \\
\hline Muscle injuries, $\mathrm{n}$ (\% of all injuries) & $283(39 \%)$ & $303(44 \%)$ & $125(45 \%)$ & $95(33 \%)$ & $266(35 \%)$ \\
\hline Muscle injury rate $/ 1000$ hours $(95 \% \mathrm{Cl})$ & 9.7 (8.6 to 10.9$)$ & $10.6(9.5$ to 11.9$)$ & 11.4 (9.6 to 13.6$)$ & 7.6 (6.2 to 9.2$)$ & $8.0(7.1$ to 9.0$)$ \\
\hline Muscle injury rate ratio $(95 \% \mathrm{Cl})$ & Reference group & 1.09 (0.93 to 1.29$)$ & 1.18 (0.95 to 1.45$)$ & 0.78 (0.62 to 0.98$)$ & $0.82(0.69$ to 0.97$)$ \\
\hline \multicolumn{6}{|l|}{$<90$ min exposure in previous match } \\
\hline Total match exposure, hours & 14395 & 13135 & 4810 & 5107 & 14411 \\
\hline All injuries, $n$ & 375 & 357 & 106 & 131 & 384 \\
\hline Total injury rate/1000 hours $(95 \% \mathrm{Cl})$ & 26.1 (23.5 to 28.8$)$ & $27.2(24.5$ to 30.1$)$ & $22.0(18.2$ to 26.7$)$ & 25.6 (21.6 to 30.4$)$ & 26.6 (24.1 to 29.5$)$ \\
\hline Total injury rate ratio $(95 \% \mathrm{Cl})$ & Reference group & 1.04 (0.90 to 1.21$)$ & 0.85 (0.68 to 1.05$)$ & $0.98(0.81$ to 1.20$)$ & 1.02 (0.89 to 1.18$)$ \\
\hline Muscle injuries, $n$ (\% of all injuries) & $152(41 \%)$ & $138(39 \%)$ & $32(30 \%)$ & $44(34 \%)$ & $123(32 \%)$ \\
\hline Muscle injury rate $/ 1000$ hours $(95 \% \mathrm{Cl})$ & $10.6(9.0$ to 12.4$)$ & 10.5 (8.9 to 12.4$)$ & 6.7 (4.7 to 9.4$)$ & $8.6(6.4$ to 11.6$)$ & 8.5 (7.2 to 10.2$)$ \\
\hline Muscle injury rate ratio $(95 \% \mathrm{Cl})$ & Reference group & 0.99 (0.79 to 1.25$)$ & $0.63(0.43$ to 0.92$)$ & 0.82 (0.58 to 1.14$)$ & 0.81 (0.64 to 1.03$)$ \\
\hline
\end{tabular}

Injury rate ratios were analysed between short-term match congestion groups using $\leq 3$ days as reference.

Values in bold indicate significant difference compared with $\leq 3$ days.

* Long-term match congestion denotes total registered match exposure for the individual during the 30 days prior to an observation. 
congestion groups. These cut-offs were chosen in conjunction to the number of full match exposures ( 1.5 hours/full match) that were closest to the 25 th percentiles. General and muscle injury rates in these groups and RRs between the groups were analysed with Poisson regressions using match exposure hours as an offset. In these analyses, long-term match congestion was included as the independent variable, with high ( $>7.5$ hours) serving as reference category, while number of injuries and muscle injuries were used as dependent variables in separate analyses.

Second, to investigate whether associations between short-term match congestion and muscle injury rates during matches were dependent on long-term match congestion, these associations were analysed in each of the long-term match congestion groups separately. Third, associations between short-term match congestion and injury rate to specific muscle groups (hip/groin, thigh and lower leg) were analysed. These analyses were also made with Poisson regressions including short-term match congestion as the independent variable, with $\leq 3$ days serving as the reference category, and using match exposure hours as an offset. All results are presented with RRs including 95\% CI.

All tests were 2-sided, and the significance level was set at $\mathrm{p}<0.05$.

\section{RESULTS}

A total of 133170 individual match observations were included in the analyses adding up to a total of 166433 match hours. During this match exposure, 4083 injuries were reported including 1561 muscle injuries (table 2). This yielded a total injury rate of 24.5/1000 match hours and a muscle injury rate of 9.4 injuries/1000 match hours.

\section{Associations between short-term match congestion and injury rates}

There were no associations between total match injury rates and short-term match congestion. Muscle injury rates were 21\% lower when there were 6 days, and 19\% lower when there were 7-10 days compared with when there were $\leq 3$ days between matches. However, no differences were found when matches were separated by 4 or 5 days compared with $\leq 3$ (table 2 and figure 1).

\section{Influence of previous match exposure}

When the observations were grouped based on the duration of the previous match exposure ( $<90 \mathrm{~min}$ and $\geq 90 \mathrm{~min}$ ), similar RRs, with 18\%-22\% fewer muscle injuries when matches were separated by 6 or $7-10$ days compared with $\leq 3$, were shown in both groups. However, these differences were significant only if the duration of the previous exposure had been $\geq 90 \mathrm{~min}$. In addition, a significant $37 \%$ reduction in muscle injury rate were shown if matches were separated by 5 days compared with 3 or less if the duration of the previous match exposure were $<90 \mathrm{~min}$ (table 2 ).

\section{Influence of long-term match congestion}

No differences between general or muscle injury rates were shown when $\leq 4.5$ hours or $>4.5$ to $\leq 7.5$ hours were compared with $>7.5$ hours (table 3 ). When association between shortterm match congestion and muscle injury rates was analysed separately in these groups, similar RRs were observed with 17\%-31\% lower muscle injury rates when there were 6 and 7-10 days between matches compared with $\leq 3$ days in all long-term match congestion groups. These differences were, however, not significant except for when 7-10 days
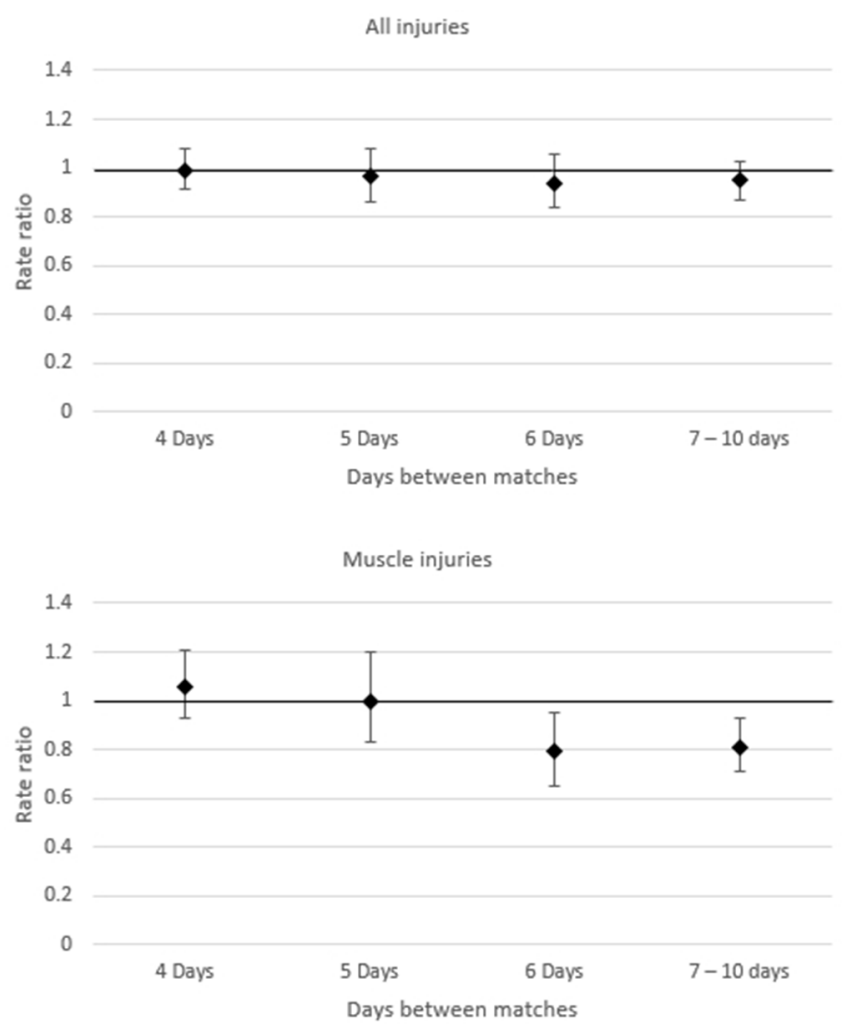

Figure 1 Analysis of the association between short-term match congestion and injury rates. Injury rate (injuries/1000 hours) ratios with corresponding $95 \%$ confidence intervals $(\mathrm{Cl})$ are presented using $\leq 3$ days between matches as reference category. Rate ratio $>1.0$ indicates a higher injury incidence. Rate ratio $<1.0$ indicates lower injury incidence. Significant difference is present if $\mathrm{Cls}$ do not include 1.0.

was compared with $\leq 3$ days in the medium long-term match congestion group (table 3 and figure 2).

\section{Injuries to specific muscle groups}

Hip/groin, thigh and lower leg muscle injuries were analysed separately in match observations where the duration of the previous match exposure was $\geq 90 \mathrm{~min}$. These analyses showed similar patterns for hip/groin and thigh injuries with $18 \%-22 \%$ fewer injuries when matches were separated by 6 or $7-10$ days compared with $\leq 3$. These differences were, however, not statistically significant. Non-significantly fewer lower leg injuries were also shown when there were 6 or $7-10$ days between matches compared with $\leq 3$. This reduction was larger than for other muscle groups after 6 days (33\%) while it was smaller after 7-10 days (13\%). In addition, statistically significant more hip/groin muscle injuries were observed when matches were separated by 5 days compared with $\leq 3$ (table 4 ).

\section{DISCUSSION}

The main finding of the current study involving more than 130 000 match observations was that the risk for professional football players to experience a muscle injury during a match was significantly associated with short-term match congestion.

\section{Lower muscle injury rates after 6 days}

If players were allowed 6 or 7-10 days between their match exposures, the muscle injury rate was about $20 \%$ lower than if they had $\leq 3$ days. Similar, but non-significant, RRs following 6 days or 7-10 days between matches were shown for hip/groin, thigh and lower leg muscle injuries. This is highly relevant for all medical practitioners working in professional football, and 
Table 3 Characteristics of individual match observations following a full match exposure ( $\geq 90 \mathrm{~min}$ ) subgrouped based on long-term match congestion and categorised depending on short-term match congestion

\begin{tabular}{|c|c|c|c|c|c|}
\hline & \multicolumn{5}{|c|}{ Long-term match congestion (total registered match exposure for the individual during 30 days) } \\
\hline & \multicolumn{2}{|l|}{ Low ( $\leq 4.5$ hours) } & \multicolumn{2}{|l|}{$\begin{array}{l}\text { Medium } \\
\text { ( }>4.5 \text { to } \leq 7.5 \text { hours })\end{array}$} & High (>7.5 hours) \\
\hline Observations, n (\%) & $16652(18 \%)$ & & $41092(48 \%)$ & & $27416(33 \%)$ \\
\hline Total match exposure, hours & 20955 & & 55537 & & 38083 \\
\hline Mean match exposure time/observation, minutes (SD) & $75.5(26.8)$ & & $81.1(21.0)$ & & $83.3(18.2)$ \\
\hline All injuries, $\mathrm{n}$ & 506 & & 1346 & & 878 \\
\hline Total injury rate/1000 hours $(95 \% \mathrm{Cl})$ & 24.1 (22.1 to 26.3$)$ & & $24.2(23.0$ to 25.6$)$ & & 23.1 (21.6 to 24.6$)$ \\
\hline Total injury rate ratio $(95 \% \mathrm{Cl})$ & 1.05 (0.94 to 1.17$)$ & & 1.05 (0.97 to 1.14$)$ & & Reference group \\
\hline Muscle injuries, $\mathrm{n}$ (\% of all injuries) & $210(42 \%)$ & & $520(39 \%)$ & & $342(39 \%)$ \\
\hline Muscle injury rate/ 1000 hours $(95 \% \mathrm{Cl})$ & $10.0(8.8$ to 11.5$)$ & & $9.4(8.6$ to 10.2$)$ & & $9.0(8.1$ to 10.0$)$ \\
\hline \multirow[t]{3}{*}{ Muscle injury rate ratio $(95 \% \mathrm{Cl})$} & $1.12(0.94$ to 1.33$)$ & & $1.04(0.91$ to 1.20$)$ & & Reference group \\
\hline & \multicolumn{5}{|c|}{ Short-term match congestion (number of days between match exposures) } \\
\hline & $\leq 3$ days & 4 days & 5 days & 6 days & $7-10$ days \\
\hline \multicolumn{6}{|l|}{ Low long-term match congestion ( $\leq 4.5$ hours)* } \\
\hline Observations, $\mathrm{n}(\%)$ & $2996(18 \%)$ & $3049(18 \%)$ & $1222(7 \%)$ & $1991(12 \%)$ & $7394(44 \%)$ \\
\hline Total match exposure, hours & 3504 & 3617 & 1544 & 2633 & 9657 \\
\hline Mean match exposure time/observation, minutes (SD) & $70.2(30.4)$ & $71.2(29.8)$ & $75.8(26.8)$ & $79.4(23.1)$ & $78.4(24.2)$ \\
\hline Mean long-term match congestion, hours (SD) & $3.5(1.0)$ & $3.5(1.0)$ & $3.5(1.0)$ & $3.6(1.0)$ & $3.6(1.0)$ \\
\hline Muscle injuries, $n$ (\% of all injuries) & $37(45 \%)$ & $47(48 \%)$ & $24(55 \%)$ & $23(36 \%)$ & $79(36 \%)$ \\
\hline Muscle injury rate/1000 hours $(95 \% \mathrm{Cl})$ & $10.6(7.7$ to 14.6$)$ & $13.0(9.8$ to 17.3$)$ & 15.5 (10.4 to 23.2$)$ & 8.7 (5.8 to 13.1$)$ & 8.2 (6.6 to 10.2$)$ \\
\hline Muscle injury rate ratiot $(95 \% \mathrm{Cl})$ & Reference group & $1.23(0.80$ to 1.89$)$ & 1.47 (0.88 to 2.46$)$ & 0.83 (0.49 to 1.39$)$ & 0.77 (0.52 to 1.14$)$ \\
\hline \multicolumn{6}{|l|}{ Medium long-term match congestion ( $>4.5$ to $\leq 7.5$ hours) * } \\
\hline Observations, n (\%) & $9275(23 \%)$ & $9644(23 \%)$ & $3942(10 \%)$ & $4955(12 \%)$ & $13276(32 \%)$ \\
\hline Total match exposure, hours & 12077 & 12797 & 5350 & 6887 & 18427 \\
\hline Mean match exposure time/observation, minutes (SD) & $78.1(24.1)$ & $79.6(22.9)$ & $81.4(20.1)$ & $83.4(18.0)$ & $83.3(18.0)$ \\
\hline Mean long-term match congestion, hours (SD) & $6.5(0.9)$ & $6.5(0.9)$ & $6.5(0.9)$ & $6.4(0.9)$ & $6.3(0.8)$ \\
\hline Muscle injuries, $n$ (\% of all injuries) & $124(40 \%)$ & $131(44 \%)$ & $65(46 \%)$ & $53(33 \%)$ & $147(34 \%)$ \\
\hline Muscle injury rate/1000 hours $(95 \% \mathrm{Cl})$ & $10.3(8.6$ to 12.2$)$ & $10.2(8.6$ to 12.1$)$ & $12.1(9.5$ to 15.5$)$ & $7.7(5.9$ to 10.1$)$ & $8.0(6.8$ to 9.4$)$ \\
\hline Muscle injury rate ratiot $(95 \% \mathrm{Cl})$ & Reference group & $1.00(0.78$ to 1.27$)$ & 1.18 (0.88 to 1.60$)$ & 0.75 (0.54 to 1.03 ) & 0.78 (0.61 to 0.99$)$ \\
\hline \multicolumn{6}{|l|}{ High long-term match congestion ( $>7.5$ hours)* } \\
\hline Observations, $\mathrm{n}(\%)$ & $9907(36 \%)$ & $8666(32 \%)$ & $2899(11 \%)$ & $2174(8 \%)$ & $3770(14 \%)$ \\
\hline Total match exposure, hours & 13553 & 12084 & 4050 & 3058 & 5338 \\
\hline Mean match exposure time/observation, minutes (SD) & $82.1(19.9)$ & $83.7(18.2)$ & $83.8(16.7)$ & $84.4(16.3)$ & $85.0(15.4)$ \\
\hline Mean long-term match congestion, hours (SD) & $9.4(1.1)$ & $9.3(1.0)$ & $9.2(1.0)$ & $9.0(0.9)$ & $8.8(0.8)$ \\
\hline Muscle injuries, $n$ ( $\%$ of all injuries) & $122(37 \%)$ & $125(43 \%)$ & $36(39 \%)$ & $19(29 \%)$ & $40(38 \%)$ \\
\hline Muscle injury rate/1000 hours $(95 \% \mathrm{Cl})$ & $9.0(7.5$ to 10.7$)$ & 10.3 (8.7 to 12.3$)$ & $8.9(6.4$ to 12.3$)$ & 6.2 (4.0 to 9.7$)$ & 7.5 (5.5 to 10.2$)$ \\
\hline Muscle injury rate ratiot $(95 \% \mathrm{Cl})$ & Reference group & $1.15(0.90$ to 1.47$)$ & 0.99 (0.68 to 1.43$)$ & 0.69 (0.43 to 1.12$)$ & 0.83 (0.58 to 1.19$)$ \\
\hline
\end{tabular}

Values in bold indicate significant difference compared with $\leq 3$ days.

*Long-term match congestion denotes total registered match exposure for the individual during 30 days.

†Injury rate ratios were analysed between long-term match congestion groups using $>7.5$ hours as reference group and between short-term match congestion groups using $\leq 3$ days as reference.

is in line with most previous studies in the field. ${ }^{9-12}$ Although the associations between match congestion and injury rates were found to be statistically significant it should be acknowledged that the individual player is still unlikely to suffer an injury in a given match even when facing a congested match schedule. In addition, there were no differences in injury rates in matches that were played $\leq 3$ days after a previous match exposure compared with 4 or even 5 days. This finding is interesting since a rule change, which would allow teams to have at least 4 days to recover between their matches, has recently been suggested. ${ }^{15}$ The main argument for introducing such a rule is that it could potentially reduce injuries in the sport, but the current study suggests that a 4th day between matches would have limited impact on injury rates in professional football.

One possibility for teams to reduce short-term match congestion for their players is a planned player rotation strategy. The analyses of players who had less than 90 min of exposure in their previous match showed that they had fewer muscle injuries when matches were separated by 5 days compared with 3 while there were no differences in injury rates after 5 compared with 3 days for players who had $90 \mathrm{~min}$ or more exposure in their previous match. This finding will offer some support that teams could potentially reduce the effect of short-term match congestion with the use of player rotation and substitutions.

Traditional player rotation will, however, not be sufficient to completely protect all players from potentially harmful match congestion. One way to make player rotation strategies more efficient could be to focus on those players most in need of recovery, and those most affected by match congestion. However, to date, there is no consensus concerning the underlying mechanisms for the associations between match congestion and injury rates in professional football, and several different 

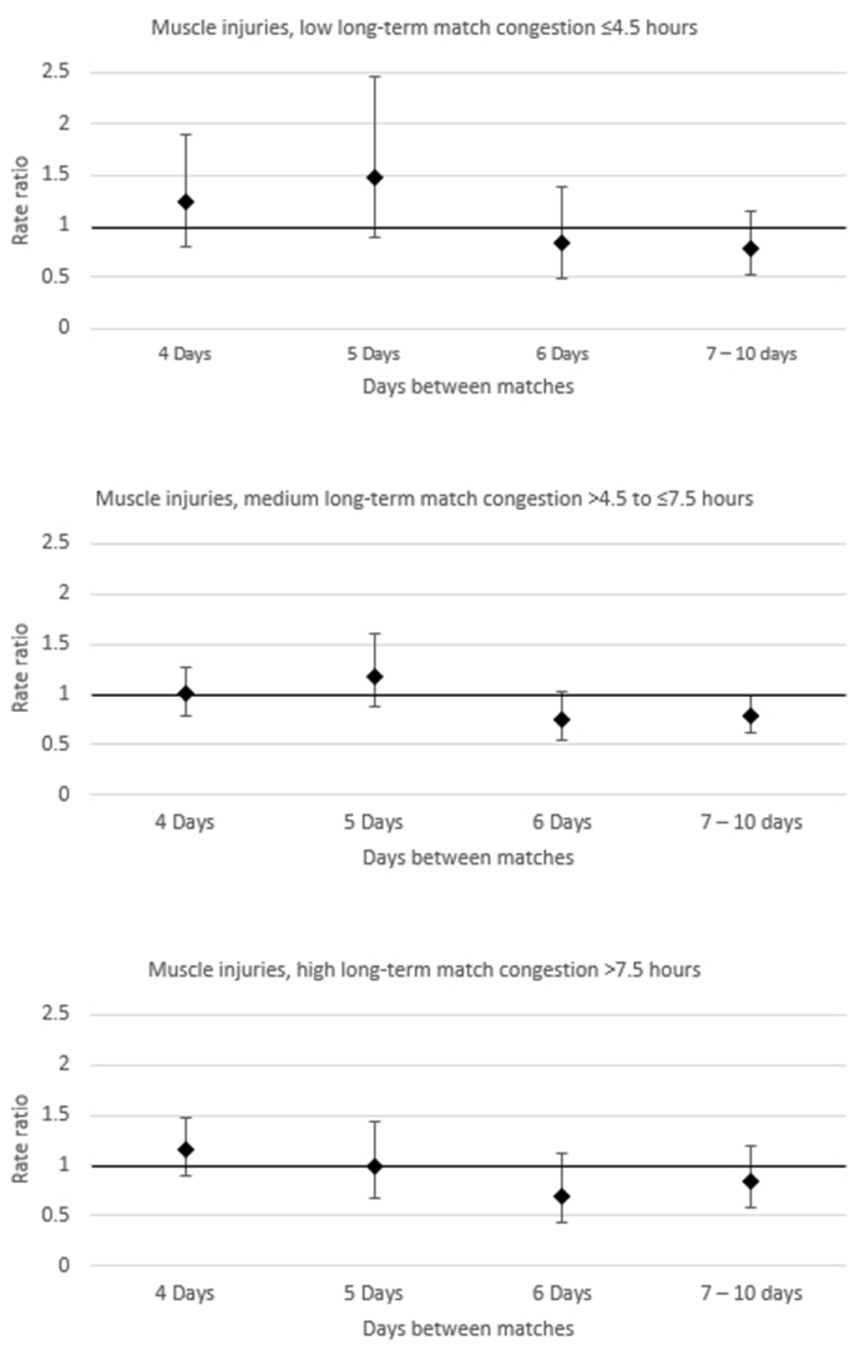

Figure 2 Analysis of the association between short-term match congestion and muscle injury rates in groups based on long-term match congestion. Injury rate (injuries/1000 hours) ratios with corresponding $95 \%$ confidence intervals $(\mathrm{Cl})$ are presented using $\leq 3$ days between matches as the reference category. Rate ratio $>1.0$ indicates higher injury incidence. Rate ratio $<1.0$ indicates lower injury incidence. Significant difference is present if $\mathrm{Cls}$ do not include 1.0. mechanisms have been suggested. ${ }^{2}$ Subjective measurements of well-being have been suggested to be sensitive in measuring both the load of athletes but also their reaction to this load and could potentially be useful for clinicians to identify players in need of rest. ${ }^{16}$ Whether or not subjective measurements of wellbeing are also associated with injury risk needs to be addressed in future studies. It should, however, also be acknowledged that while subjective measurements may be able to describe training load, the best way to implement them are still under discussion ${ }^{17}$ and possible effects of recall bias as well as questionnaire fatigue need to be considered when interpreting the results. ${ }^{17} 18$

\section{Muscle fatigue: a potential mechanism}

A possible mechanism explaining the higher match muscle injury rates during periods of match congestion is remaining muscular fatigue following a match. Signs of muscular fatigue after a football match or similar physical activity have been shown to remain for up to 72 hours in several studies. ${ }^{3-6}$ These studies have all shown significant effects on muscle performance throughout 72 hours of recovery after a match. In addition, objective signs of muscle damage and inflammatory reaction, ${ }^{3-6}$ as well as higher subjective ratings of muscle soreness, ${ }^{56}$ have also been demonstrated.

Studies have also shown that the biomechanical properties of muscles may change during and immediately after a match-simulating exercise resulting in lower maximum force production, lower muscle torque rate and changes in muscle activation patterns. ${ }^{19-22}$ These changes may alter player movements, subjecting specific muscles to activity levels/patterns that they are not accustomed to and thereby exposing players to higher injury risk. It is, however, not known how long it takes for these factors to return to preactivity levels.

\section{Other potential mechanisms}

A recent consensus statement about load in sports argues that load of athletes is a complex phenomenon which consists of physiological as well as psychological aspects. ${ }^{23}$

In football, studies have indicated that mental fatigue decreases the decision-making ability of players in match play situations, ${ }^{8}$ as well as their physical and technical performance immediately after completing a mentally fatiguing exercise. ${ }^{24}$ It is plausible that a player with reduced decision-making abilities as well as physical and technical abilities will face more high-risk situations during a match such as receiving a tackle or being forced to sprint. There

Table 4 Muscle injury rate in different muscle groups following a full match exposure ( $\geq 90 \mathrm{~min}$ ) categorised depending on short-term match congestion

\begin{tabular}{|c|c|c|c|c|c|}
\hline & \multicolumn{5}{|c|}{ Short-term match congestion (number of days between match exposures) } \\
\hline & $\leq 3$ days & 4 days & 5 days & 6 days & $7-10$ days \\
\hline \multicolumn{6}{|l|}{ Hip/groin muscle injury rates } \\
\hline Hip/groin muscle injuries, $n$ (\% of injuries) & $71(10 \%)$ & $76(11 \%)$ & $43(16 \%)$ & $24(8 \%)$ & $67(9 \%)$ \\
\hline Hip/groin muscle injury rate/1000 hours $(95 \% \mathrm{Cl})$ & $2.4(1.9$ to 3.1$)$ & $2.7(2.1$ to 3.3$)$ & $3.9(2.9$ to 5.3$)$ & $1.9(1.3$ to 2.8$)$ & $2.0(1.6$ to 2.5$)$ \\
\hline Hip/groin muscle injury rate ratio $(95 \% \mathrm{Cl})$ & Reference group & 1.09 (0.79 to 1.51$)$ & 1.61 (1.10 to 2.35$)$ & 0.78 (0.49 to 1.24$)$ & $0.82(0.59$ to 1.15$)$ \\
\hline \multicolumn{6}{|l|}{ Thigh muscle injury rates } \\
\hline Thigh muscle injuries, $\mathrm{n}$ (\% of injuries) & $171(24 \%)$ & $174(25 \%)$ & $65(23 \%)$ & $58(20 \%)$ & $161(21 \%)$ \\
\hline Thigh muscle injury rate/ 1000 hours $(95 \% \mathrm{Cl})$ & 5.9 (5.1 to 6.8$)$ & $6.1(5.3$ to 7.1$)$ & 5.9 (4.7 to 7.6$)$ & 4.6 (3.6 to 6.0$)$ & $4.8(4.1$ to 5.6$)$ \\
\hline Thigh muscle injury rate ratio $(95 \% \mathrm{Cl})$ & Reference group & 1.04 (0.84 to 1.28$)$ & $1.01(0.76$ to 1.35$)$ & 0.79 (0.58 to 1.06$)$ & 0.82 (0.66 to 1.02$)$ \\
\hline \multicolumn{6}{|l|}{ Lower leg muscle injury rates } \\
\hline Lower leg muscle injuries, $n$ (\% of injuries) & $31(4 \%)$ & $43(6 \%)$ & $13(5 \%)$ & $9(3 \%)$ & $31(4 \%)$ \\
\hline Lower leg muscle injury rate/1000 hours $(95 \% \mathrm{Cl})$ & $1.1(0.7$ to 1.5$)$ & 1.5 (1.1 to 2.0$)$ & $1.2(0.7$ to 2.0$)$ & $0.7(0.4$ to 1.4$)$ & $0.9(0.7$ to 1.3$)$ \\
\hline Lower leg muscle injury rate ratio $(95 \% \mathrm{Cl})$ & Reference group & 1.42 (0.89 to 2.25$)$ & 1.12 (0.58 to 2.13$)$ & 0.67 (0.32 to 1.41$)$ & 0.87 (0.53 to 1.43$)$ \\
\hline
\end{tabular}

Injury rate ratios were analysed between short-term match congestion groups using $\leq 3$ days as reference.

Values in bold indicate significant difference compared with $\leq 3$ days. 
are, however, no studies on the development of mental fatigue following a match and during the subsequent recovery period and no studies investigating mental fatigue during a period of match congestion.

\section{Relationship between long-term and short-term match congestion}

Long-term match congestion has been shown to increase injury rates in professional football. ${ }^{9} 1112$ It has also been shown that muscle fatigue and signs of muscle damage following a match are greater when the recovery time between matches has been short, suggesting an accumulation of fatigue. ${ }^{5}$ Muscle fatigue and muscle damage could potentially make players more vulnerable to shortterm match congestion. On the other hand, in elite rugby, high long-term (chronic) workload has been shown to protect athletes from increased injury risk during short-term match congestion. ${ }^{25}$ In the present study, no associations between long-term match congestion and injury rates were identified. While this is in contrast with a previous study using a shorter, more intense period of match congestion (6 matches all separated by 3 days), ${ }^{11}$ it is in line with another study using a more similar definition of high match congestion ( 8 matches in 28 days). ${ }^{13}$

In addition, when match observations were separated into low, medium and high long-term match congestion groups, similar RRs, with lower muscle injury rates after 6 or more days since the last match exposure compared with less, emerged in all groups. This indicates that the associations between short-term match congestion and muscle injury rates were not dependent on players' long-term match congestion.

\section{Methodological considerations}

The study finds merit in that it follows international consensus about how to conduct epidemiological studies in football. The major strength is the large and homogenous cohort of professional football teams from several countries, with more than 130000 individual match observations, which allows for a more detailed categorisation of the different match congestion variables than those in previous studies. ${ }^{10-13}$ It should, however, be acknowledged that even though the study included a large cohort, there were still relatively few injuries included in some groups in the subanalyses and the findings from these should therefore be interpreted with caution. The fact that most of the RRs in these subanalyses were found to be non-significant may also suggest a lack of statistical power.

A second limitation with the study is that the load during training sessions was not measured. In addition, match congestion, as used in the current study, only measures the interval with which matches were played and does not take into account the activity level of players during these matches. There are several factors that may influence the activity level of players during matches. Previous studies have, for example, shown that playing formation, possession of the ball and the current score line of the match will influence players' activity level. ${ }^{26-28}$ It is reasonable to believe that players' activity level during match-free days as well as their activity level during specific matches might contribute to their level of fatigue, which could potentially be of importance for their injury risk. It should also be acknowledged that match-related factors that influence the activity profile of participating players as discussed above, and which have not been taken into consideration in the present study, may also have a direct effect on the risk of sustaining an injury during a match exposure.

Furthermore, only external load (the amount of work performed by the athlete) was considered, ${ }^{29}$ while internal load (relative physiological and psychological stress imposed on the athlete) was not included. A concern with measurements of external load is that they may only measure specific aspects of the load that athletes are exposed to, that is, match congestion in the current study. However, internal load measures are also needed to capture the full picture of the load put on athletes and how they react to this load. This issue is supported by data from cricket where measurements of internal load have been shown to be a better predictor of injuries than external load. ${ }^{30}$ Studies with more detailed information of the activity level during all training sessions as well as matches, and with a focus on the internal load might therefore be able to contribute further to the understanding of how load is associated with injury rates in professional football.

Finally, observational studies are always at risk of confounding factors. In the present study, some possible confounders have been considered by several separate analyses of different subgroups based on previous exposures. However, the risk of remaining confounders should not be disregarded. One possible confounder is that the selection of players before matches may be influenced by the short-term match congestion. This would mean that there, to some extent, could be different players included in the different short-term match congestion groups that were analysed which could potentially influence the results. A second possible confounder is that players' long-term match congestion could be influenced by a previous injury. Even though we used an exclusion criteria saying that players could not have been absent due to injury during the last 30 days prior to a match, it is still possible that an injury absence prior to those 30 days could affect players' long-term match congestion. It is plausible that players who have recently been absent due to injury would have lower long-term match congestion compared with players who have not been previously injured. A previous injury is an established risk factor for sustaining a secondary injury ${ }^{31}$ and previous injuries could thus potentially influence our results.

\section{CONCLUSION}

In this study of male professional football players, there were no match congestion-related differences in total match injury rates, but muscle injury rates during matches were lower when players were given at least 6 days between their match exposures.

What are the findings?

- There were no differences in total injury rates depending on the number of days separating two matches.

- Muscle injury rates were lower after $\geq 6$ days between matches compared with $\leq 3$ days.

- There were no differences in total or muscle injury rates depending on players' long-term match congestion.

How might it impact on clinical practice in the future?

- These findings may help guide technical staff in football teams when making decisions about players' match congestion suggesting that player rotation may be of importance to avoid injuries even when matches are separated by 5 days.

- Remaining effects of a football match needs to be considered and monitored, and possible recovery strategies may be of importance up to 6 days following a match. 
Acknowledgements The authors would like to thank the participating clubs (including contact persons, coaching staff, medical staff and all players) for their participation in the study. The authors would also like to thank Henrik Magnusson (HM) for insightful comments and help with statistical analyses. The Football Research Group is established in Linköping, Sweden, in collaboration with Linköping University and through grants from the Union of European Football Associations, Swedish Football Association, Football Association Premier League Limited and Swedish National Centre for Research in Sports.

Contributors HB was responsible for the conception and design of the study. HB and $\mathrm{HM}$ conducted the analyses, which were planned and checked with $\mathrm{MH}$, JE and MW. All authors contributed to the interpretation of the findings and had full access to all data. HB wrote the first draft of the paper, which was critically revised by JE, $\mathrm{MW}$ and $\mathrm{MH}$. The final manuscript was approved by all authors. MH is the study guarantor.

Funding This study was funded by grants from the Union of European Football Associations and the Swedish National Centre for Research in Sports.

Competing interests None declared.

Patient consent Obtained.

Ethics approval The study protocol was approved by the UEFA Football Development Division and the UEFA Medical Committee.

Provenance and peer review Not commissioned; externally peer reviewed.

(c) Article author(s) (or their employer(s) unless otherwise stated in the text of the article) 2018. All rights reserved. No commercial use is permitted unless otherwise expressly granted.

\section{REFERENCES}

1 Ekstrand J, Hägglund M, Kristenson K, et al. Fewer ligament injuries but no preventive effect on muscle injuries and severe injuries: an 11-year follow-up of the UEFA Champions League injury study. Br I Sports Med 2013;47:732-7.

2 Nédélec M, McCall A, Carling C, et al. Recovery in soccer: part I - post-match fatigue and time course of recovery. Sports Med 2012;42:997-1015.

3 Ascensão A, Rebelo A, Oliveira E, et al. Biochemical impact of a soccer match analysis of oxidative stress and muscle damage markers throughout recovery. Clin Biochem 2008:41:841-51.

4 Fatouros IG, Chatzinikolaou A, Douroudos II, et al. Time-course of changes in oxidative stress and antioxidant status responses following a soccer game. J Strength Cond Res 2010;24:3278-86

5 Mohr M, Draganidis D, Chatzinikolaou A, et al. Muscle damage, inflammatory, immune and performance responses to three football games in 1 week in competitive male players. Eur J Appl Physiol 2016;116:179-93.

6 Nedelec M, McCall A, Carling C, et al. The influence of soccer playing actions on the recovery kinetics after a soccer match. J Strength Cond Res 2014;28:1517-23.

7 Coutts AJ. Fatigue in football: it's not a brainless task!. J Sports Sci 2016;34:1296.

8 Smith MR, Zeuwts L, Lenoir M, et al. Mental fatigue impairs soccer-specific decisionmaking skill. J Sports Sci 2016;34:1297-304.

9 Bengtsson H, Ekstrand J, Hägglund M. Muscle injury rates in professional football increase with fixture congestion: an 11-year follow-up of the UEFA Champions League injury study. Br J Sports Med 2013;47:743-7.

10 Dupont G, Nedelec M, McCall A, et al. Effect of 2 soccer matches in a week on physical performance and injury rate. Am J Sports Med 2010;38:1752-8.
11 Dellal A, Lago-Peñas C, Rey E, et al. The effects of a congested fixture period on physical performance, technical activity and injury rate during matches in a professional soccer team. Br J Sports Med 2015;49:390-4.

12 Carling C, McCall A, Le Gall F, et al. The impact of short periods of match congestion on injury risk and patterns in an elite football club. Br J Sports Med 2016;50:764-8.

13 Carling C, Le Gall F, Dupont G. Are physical performance and injury risk in a professional soccer team in match-play affected over a prolonged period of fixture congestion? Int J Sports Med 2012;33:36-42.

14 Hägglund $M$, Waldén $M, B a h r ~ R$, et al. Methods for epidemiological study of injuries to professional football players: developing the UEFA model. Br I Sports Med 2005;39:340-6.

15 Verheijen R. Study on recovery days: World Football Academy, 2012. http:// worldfootballacademy.com/wp-content/uploads/2012/05/WFA_Study-on-recoverydays.pdf (accessed 8 Sep 2016).

16 Saw AE, Main LC, Gastin PB. Monitoring the athlete training response: subjective selfreported measures trump commonly used objective measures: a systematic review. Br J Sports Med 2016;50:281-91.

17 Saw AE, Main LC, Gastin PB. Monitoring athletes through self-report: factors influencing implementation. J Sports Sci Med 2015;14:137-46.

18 Borresen J, Lambert MI. The quantification of training load, the training response and the effect on performance. Sports Med 2009;39:779-95.

19 Marshall PW, Lovell R, Jeppesen GK, et al. Hamstring muscle fatigue and central motor output during a simulated soccer match. PLoS One 2014;9:e102753.

20 Small K, McNaughton L, Greig M, et al. The effects of multidirectional soccer-specific fatigue on markers of hamstring injury risk. J Sci Med Sport 2010;13:120-5.

21 Jones RI, Ryan B, Todd Al. Muscle fatigue induced by a soccer match-play simulation in amateur Black South African players. J Sports Sci 2015;33:1305-11.

22 Stutzig N, Siebert T. Muscle force compensation among synergistic muscles after fatigue of a single muscle. Hum Mov Sci 2015:42:273-87.

23 Soligard T, Schwellnus M, Alonso JM, et al. How much is too much? (Part 1) International Olympic Committee consensus statement on load in sport and risk of injury. Br J Sports Med 2016;50:1030-41.

24 Smith MR, Coutts AJ, Merlini M, et al. Mental Fatigue Impairs Soccer-Specific Physical and Technical Performance. Med Sci Sports Exerc 2016;48:267-76.

25 Hulin BT, Gabbett TJ, Caputi P, et al. Low chronic workload and the acute:chronic workload ratio are more predictive of injury than between-match recovery time: a two-season prospective cohort study in elite rugby league players. $\mathrm{Br} / \mathrm{Sports} \mathrm{Med}$ 2016;50:1008-12.

26 Bradley PS, Noakes TD. Match running performance fluctuations in elite soccer: indicative of fatigue, pacing or situational influences? I Sports Sci 2013;31:1627-38.

27 Bradley PS, Carling C, Archer D, et al. The effect of playing formation on high-intensity running and technical profiles in English FA Premier League soccer matches. I Sports Sci 2011;29:821-30.

28 da Mota GR, Thiengo CR, Gimenes SV, et al. The effects of ball possession status on physical and technical indicators during the 2014 FIFA World Cup Finals. J Sports Sci 2016:34:493-500

29 Windt J, Gabbett TJ. How do training and competition workloads relate to injury? The workload-injury aetiology model. Br J Sports Med 2017;51:428-35.

30 Hulin BT, Gabbett TJ, Blanch P, et al. Spikes in acute workload are associated with increased injury risk in elite cricket fast bowlers. Br J Sports Med 2014:48:708-12.

31 Hägglund $M$, Waldén $M$, Ekstrand J. Injury recurrence is lower at the highest professional football level than at national and amateur levels: does sports medicine and sports physiotherapy deliver? Br J Sports Med 2016;50:751-8. 